PREPARED FOR THE U.S. DEPARTMENT OF ENERGY, UNDER CONTRACT DE-AC02-76CH03073

PPPL-3618

PPPL-3618

UC-70

Regime for a Self-ionizing Raman Laser Amplifier

by

D.S. Clark and N.J. Fisch

October 2001

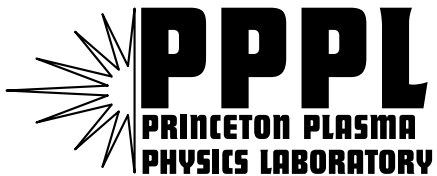

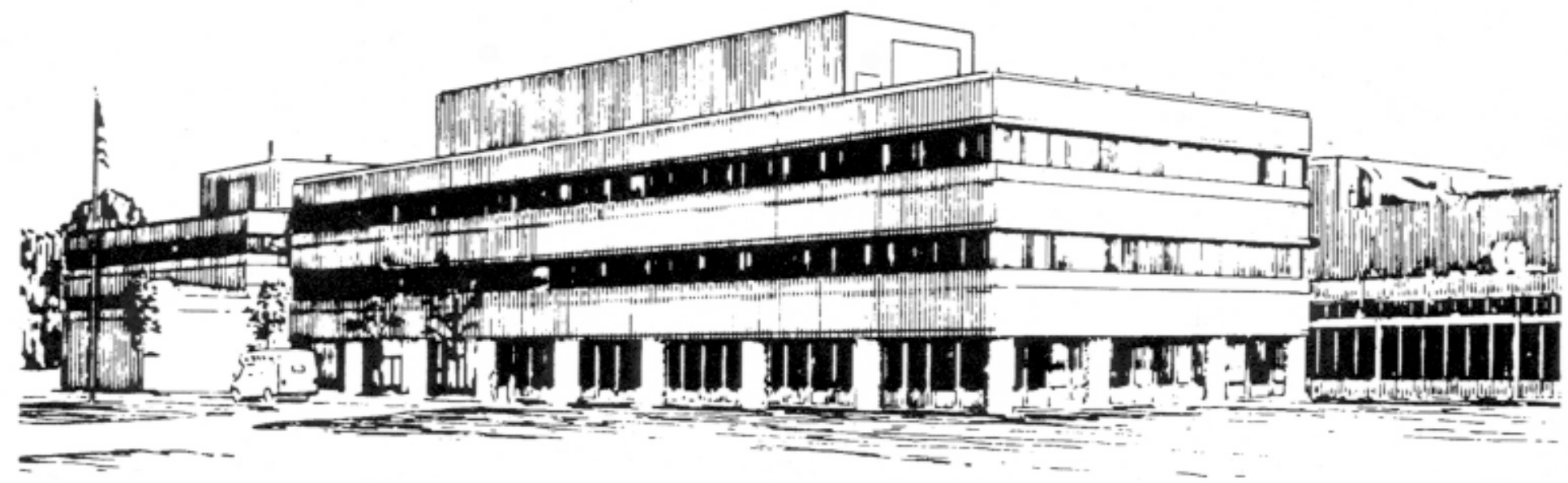

PRINCETON PLASMA PHYSICS LABORATORY PRINCETON UNIVERSITY, PRINCETON, NEW JERSEY 


\section{PPPL Reports Disclaimer}

This report was prepared as an account of work sponsored by an agency of the United States Government. Neither the United States Government nor any agency thereof, nor any of their employees, makes any warranty, express or implied, or assumes any legal liability or responsibility for the accuracy, completeness, or usefulness of any information, apparatus, product, or process disclosed, or represents that its use would not infringe privately owned rights. Reference herein to any specific commercial product, process, or service by trade name, trademark, manufacturer, or otherwise, does not necessarily constitute or imply its endorsement, recommendation, or favoring by the United States Government or any agency thereof. The views and opinions of authors expressed herein do not necessarily state or reflect those of the United States Government or any agency thereof.

\section{Availability}

This report is posted on the U.S. Department of Energy's Princeton Plasma Physics Laboratory Publications and Reports web site in FiscaL Year 2002. The home page for PPPL Reports and Publications is: http://www.pppl.gov/pub_report/

DOE and DOE Contractors can obtain copies of this report from:

U.S. Department of Energy

Office of Scientific and Technical Information

DOE Technical Information Services (DTIS)

P.O. Box 62

Oak Ridge, TN 37831

Telephone: (865) 576-8401

Fax: (865) 576-5728

Email: reports@adonis.osti.gov

This report is available to the general public from:

National Technical Information Service

U.S. Department of Commerce

5285 Port Royal Road

Springfield, VA 22161

Telephone: 1-800-553-6847 or

(703) 605-6000

Fax: (703) 321-8547

Internet: http://www.ntis.gov/ordering.htm 


\title{
Regime for a Self-ionizing Raman Laser Amplifier
}

\author{
D. S. Clark and N. J. Fisch \\ Princeton Plasma Physics Laboratory, Princeton University \\ P.O. Box 451, MS30, Princeton, New Jersey 08543
}

September 10, 2001

\begin{abstract}
Backward Raman amplification and compression at high power might occur if a long pumping laser pulse is passed through a plasma to interact resonantly with a counter-propagating short seed pulse [V. M. Malkin, et. al., Phys. Rev. Lett., 82(22):4448-4451, 1999]. One critical issue, however, is that the pump may be unacceptably depleted due to spontaneous Raman backscatter from intrinsic fluctuations in the amplifying plasma medium prior to its useful interaction with the seed. Premature backscatter may be avoided, however, by employing a gaseous medium with pump intensities too low to ionize the medium and using the intense seed to produce the plasma by rapid photoionization as it is being amplified [V. M. Malkin, et. al., Phys.Plasmas (2001)]. In addition to allowing that only rather low power pumps be used, photoionization introduces a damping of the short pulse which must be overcome by the Raman growth rate for net amplification to occur. The parameter space of gas densities, laser wavelengths, and laser intensities is surveyed to identify favorable regimes for this effect. Output laser intensities of $10^{17} \mathrm{~W} / \mathrm{cm}^{2}$ for $0.5 \mu \mathrm{m}$ radiation are found to be feasible for such a scheme using a pump of $10^{13} \mathrm{~W} / \mathrm{cm}^{2}$ and an initial seed of $5 \times 10^{14} \mathrm{~W} / \mathrm{cm}^{2}$ over an amplification length of $5.6 \mathrm{~cm}$ in hydrogen gas.
\end{abstract}




\section{Introduction}

Through the last several decades, applications such as inertial confinement fusion (ICF) [1], laser wake field particle acceleration [2], or investigations of nonlinear quantum electrodynamics [3] have driven the search for ever higher laser intensities in ever shorter pulses. In the mid-1980's the development of the chirped pulse amplification (CPA) technique enabled the remarkable increase in peak unfocused laser intensities from the then limit of $\sim 10^{9} \mathrm{~W} / \mathrm{cm}^{2}$ to the range of several $10^{12} \mathrm{~W} / \mathrm{cm}^{2}$ [4]. While the CPA technique has yielded tremendous advances in laser powers, access to yet higher powers and intensities by this process is frustrated by the requirement of impractically large diffraction gratings of the multi-meter scale and the fixed damage thresholds of these fragile, high-precision elements to ultrahigh light intensities. Nevertheless, such schemes as the fast ignitor for laser fusion (requiring $\sim 1 \mathrm{ps}$ pulses of $\sim 10^{20} \mathrm{~W} / \mathrm{cm}^{2}$ intensities) [5] or studies of photon decay by electron-positron pair production (at intensities greater than $10^{30} \mathrm{~W} / \mathrm{cm}^{2}$ ) demand powers at or beyond current limits of CPA and so motivate the search for yet more efficient as well as compact laser amplifiers capable of withstanding ultra-intense laser fluences. In this regard, the essential indestructibility of plasma under currently imaginable laser intensities particularly suggests its use as a laser amplifying element.

To meet such a need, a scheme has recently been proposed [6] for the amplification of unfocused pulses into the $10^{17} \mathrm{~W} / \mathrm{cm}^{2}$ range using backward Raman scattering in plasma slabs. In this technique, a long laser "pump" pulse of large integrated energy is collided with a narrow, oppositely-propagating "seed" pulse. The nonlinear evolution of the Raman scattering instability was found analytically to result in the scattering of the pump power into the narrow seed pulse with this backward propagating seed both amplifying and narrowing (compressing) linearly in time. A maximum efficiency for energy transfer from the pump pulse to the seed of nearly $80 \%$ should be possible with a focused seed intensity reaching on the order of $10^{5}$ times what is currently achievable.

However, the stability of the pump pulse to spontaneous Raman backscatter from intrinsic fluctuations 
in the plasma medium, which could degrade or entirely suppress the amplification effect, is of concern. Stabilization of this unwanted Raman scattering of the pump pulse might be achieved by exploiting the nonlinear narrowing of the seed as it amplifies [7]. Since the seed is rapidly narrowing in space during its nonlinear amplification, its consequent broadening in frequency renders this nonlinear amplification phase robust to "detuning" from the exact Raman resonance. The linear evolution of the premature backward scattering of the pump (during which any signal broadens as opposed to narrowing [8]), however, — without such broad spectra — would not be resilient to such deviations from resonance. The introduction of a spatial variation in the plasma frequency (i.e., density) to detune the seed from forward scattering and an opposed temporal chirp of the pump pulse to detune from backscattering would then suppress these undesired scattering mechanisms. Analytical and numerical calculations in one dimension have shown this continued nonlinear amplification of the seed pulse in the presence of slight detuning.

This analysis was performed only in the limit of a cold, collisionless fluid plasma without realistic modeling of spontaneous pump backscatter from plasma noise [9], however. Simulations with the particlein-cell (PIC) code Zohar [10], in which the effect of discreteness-induced plasma noise is included, suggest that such backscatter may be a significant concern. While the fluctuation level is significantly exaggerated in PIC codes relative to that in a real plasma (due to the far fewer particles per Debye sphere in any conceivable simulation compared to a realistic weakly coupled plasma [11]), it remains to be demonstrated convincingly that a relatively high intensity laser pump may be propagated stably through $\sim 1 \mathrm{~cm}$ of plasma of reasonable density for strong nonlinear coupling. The Raman amplification effect may be demonstrated in particle simulations using "cold" or "quiet" starts in which no fluctuations initially exist in the plasma, effectively a plasma of zero temperature; but such simulations, while including certain discrete-plasma effects, effectively sidestep the crucial question of pump stability to intrinsic noise. Indeed, electron temperatures of $\sim 200 \mathrm{eV}$ may be necessary in some implementations to avoid excessive collisional damping of the pump or seed during their propagation through the amplifier. 
To avoid the difficulties associated with propagation of the pump it has been proposed that the relatively long ( $1 \mathrm{~cm})$ plasma amplifying medium be replaced by a neutral gas of the appropriate density to satisfy the Raman resonance condition once fully ionized [12]. If a pumping pulse of intensity below the ionization threshold for the gas is used, the pumping pulse may then be propagated through the entire amplifier without the possibility of spontaneous Raman backscatter. In conjunction with a seed pulse of intensity greater than the threshold for rapid photoionization, the gas will be completely ionized to the appropriate density only at the front of the amplifying seed pulse and allow the backscatter of the pump only in this region and so only the useful amplification of the seed. Generating the plasma in situ by photoionization from the seed, however, necessarily results in an added damping of the seed. For a self-ionizing Raman amplifier to be feasible, a regime must the then be found for which not only is the pump well below and the seed well above the photoionization threshold, but also for which the nonlinear Raman growth rate of the seed exceeds it damping rate due to ionization or other effects. Total output laser intensities of $\sim 10^{17} \mathrm{~W} / \mathrm{cm}^{2}$ for $0.5 \mu \mathrm{m}$ radiation are found to be feasible for such a scheme using a pump of $10^{13} \mathrm{~W} / \mathrm{cm}^{2}$ and a seed of $5 \times 10^{14} \mathrm{~W} / \mathrm{cm}^{2}$ over an amplification length of $5.6 \mathrm{~cm}$ in hydrogen gas. This is to be compared with amplification to an equivalent output intensity in a pre-ionized plasma of length $0.35 \mathrm{~cm}$ by a pump of $10^{15} \mathrm{~W} / \mathrm{cm}^{2}$. In effect, the higher pump intensity tolerable for a plasma is traded for a longer but stabler interaction length in a neutral gas to obtain identical output intensities.

Even if spontaneous pump backscatter from noise in a pre-ionized plasma ultimately proves not to be a difficulty for the conventional amplification scheme, a self-ionizing amplifier appears still to have the advantage of simplicity. While the conventional amplifier requires the preparation (and temporary maintenance) of a plasma of a specific density, and possibly density gradient, and that the plasma be heated to $T_{e} \sim 200 \mathrm{eV}$, a self-ionizing amplifier requires only the far simpler arrangement of an amplifying chamber filled with gas to a specified uniform density. Overall, the far lower fluctuation levels and simpler handling properties of a gaseous as opposed to plasma medium suggest that a self-ionizing 
amplifier may well be favored simply from practical considerations. In addition, precursor pulses, which may disrupt backward Raman amplification in a pre-ionized plasma [13], might be eliminated due to the absence of plasma ahead of the seed pulse front.

Note that the method contemplated here is pulse compression by ionization-induced backscatter of the pump, with the ionization induced by the backscattered pulse, which is much more intense than the pump. There are other ionization effects which induce pulse shortening, primarily the erosion of

the leading edge of the pulse [14]. These effects, however, apply to high intensity femtosecond pulses traveling through ionizing plasma in the absence of a counter-propagating pump wave.

This paper demonstrates a regime in which an ionization front may be employed for coupling the pump to an amplifying seed pulse. Conditions on the pump intensity, laser polarization, working gas, and other parameters are quantified and the most favorable regime for self-ionizing amplification identified. This paper is organized as follows. Sec. 2 describes the effects and governing equations used in modeling a self-ionizing Raman amplifier. Sec. 3 describes the energetics of pulse amplification and plasma ionization which represent constraints on the possible regimes of operation for a self-ionizing Raman amplifier. Sec. 4 presents example results of the numerical integration of the model outlined in Sec. 2, and Sec. 5 offers concluding remarks and possibilities for further research.

\section{Modeling of a self-ionizing Raman amplifier}

The envelope approximation (see e.g., [15]) in one dimension is used to model the Raman backscatter interaction (see e.g., [16]) in which the two light waves $(1,2)$ are coupled by the Langmuir wave (3) 
according to the equations

$$
\begin{aligned}
& \left(\partial_{t}-v_{1} \partial_{x}-\nu_{1} / 2\right) a_{1}=K a_{2} a_{3} \\
& \left(\partial_{t}-v_{2} \partial_{x}-\nu_{2} / 2\right) a_{2}=-K a_{1} a_{3}^{*} \\
& \left(\partial_{t}-v_{3} \partial_{x}-\nu_{3} / 2\right) a_{3}=-K a_{1} a_{2}^{*} .
\end{aligned}
$$

Here $a_{i}$ is the potential envelope of each wave normalized to $m_{e} c^{2} / e$ for the light waves and $\left(\omega_{p e} / 2 \omega\right)\left(m_{e} c^{2} / e\right)$ for the Langmuir wave, $v_{i}$ the corresponding wave group velocity, and $\nu_{i}$ the wave energy damping rate. The wave coupling parameter $K$ is $\left(n_{e} / n_{c}\right)^{1 / 4} / \sqrt{2}$ for circular polarization and $\left(n_{e} / n_{c}\right)^{1 / 4} / 2$ for linear polarization with $n_{e}$ the electron density and $n_{c} \doteq m_{e} \omega^{2} / 4 \pi|e|^{2}$ the critical density corresponding to the laser frequency.

The Raman interaction requires the frequency (i.e., plasma density) and wave number matching conditions $[16]$

$$
\omega_{1}=\omega_{2}+\omega_{3} \quad \text { and } \quad \mathbf{k}_{1}=\mathbf{k}_{2}+\mathbf{k}_{3}
$$

It is assumed that the plasma is sufficiently rarefied $\left(n_{e} / n_{c} \ll 1\right)$ that the laser frequencies are approximately comparable $\omega_{1} \simeq \omega_{2} \equiv \omega \gg \omega_{3} \equiv \omega_{p}$ in Eq. (1) and that the laser phase velocities are both approximately the velocity of light in vacuum. Consequently, $k_{1,2} \simeq \omega / c$ and $k_{3} \simeq 2 k_{1,2}$ for direct backscatter. For the case of phase matching in an inhomogeneous plasma, it is assumed in the following that once the density reaches within $1 \%$ of the appropriate resonant value the coupling constant $K$ attains its full value and otherwise is zero. Also, for adequately low electron temperatures, the thermal dispersion of the Langmuir wave is negligible so that $v_{3} \simeq 0$.

For typical plasma temperatures which are well below $\sim 1 \mathrm{keV}$, Landau damping is negligible so that the damping rates in Eqs. (1) are due solely to electron-ion and electron-neutral collisions. The damping 
rates appearing in these equations are then given by [16]

$$
\nu \equiv \nu_{1,2}=\left(\frac{\omega_{p}}{\omega}\right)^{2}\left(\nu_{e i}+\nu_{n}\right) \quad \text { and } \quad \nu_{3}=\nu_{e i}+\nu_{n}
$$

with

$$
\begin{aligned}
\nu_{e i}\left[\mathrm{sec}^{-1}\right] \simeq & 2.9 \times 10^{-6} Z_{\text {eff }} n_{e}\left[\mathrm{~cm}^{-3}\right] \ln \Lambda \varepsilon[\mathrm{eV}]^{-3 / 2} \\
\nu_{n}\left[\mathrm{sec}^{-1}\right] \simeq & n_{n}\left[\mathrm{~cm}^{-3}\right] \sigma_{n}\left[\mathrm{~cm}^{2}\right] \times \\
& \begin{cases}v_{\mathrm{osc}}[\mathrm{cm} / \mathrm{sec}], & v_{\mathrm{osc}}<\alpha \\
(\alpha c)[\mathrm{cm} / \mathrm{sec}], & v_{\mathrm{osc}}>\alpha\end{cases}
\end{aligned}
$$

where $\varepsilon=T_{e}+m_{e} v_{\mathrm{osc}}^{2} / 2$ is the total particle energy, $v_{\mathrm{osc}}=c$ a its oscillation velocity in the total electric field, $n_{n}$ the number density of neutrals, $\sigma_{n} \simeq 10^{-15} \mathrm{~cm}^{2}$ the electron-neutral cross-section, $\alpha \simeq 1 / 137$ the fine structure constant, and $\ln \Lambda$ is the Coulomb logarithm $[17,18]$. In addition to damping the wave fields, collisions result in the weak background heating of the plasma according to

$$
\frac{3}{2} n_{e} \partial_{t} T_{e}=\frac{1}{8 \pi}\left[\nu\left(E_{1}^{2}+E_{2}^{2}\right)+\nu_{3} E_{3}^{2}\right]
$$

The photoionization rate of an atomic gas in an intense laser field can be modeled by the Keldysh formula [18, 19] provided $E \ll m_{e}^{2}|e|^{5} / \hbar^{4}$, or equivalently $a \ll m_{e}|e|^{6} / \hbar^{4} \omega c \simeq 0.1$ for half-micron radiation [20]. For small electric fields, $\gamma \doteq \sqrt{2 U_{I} / m_{e} c^{2}} / a \gg 1$, a multi-photon formula for the ionization rate applies,

$$
w(a) \simeq \omega N^{3 / 2}(2 \gamma)^{-2 N}
$$

where $w(a)$ is the probability of ionizing a single neutral atom per unit time, $U_{I}$ is the ionization potential, and $N \doteq 1+\operatorname{Int}\left(U_{I} / \hbar \omega\right)$ is the number of photons required to overcome that potential. For large electric fields, $\gamma \ll 1$, when the ionization rate is sufficiently rapid for the electric field to be approximated as static, a tunneling formula is accurate,

$$
w(a) \simeq 4 \Omega_{0}\left(\frac{U_{I}}{U_{H}}\right)^{5 / 2} \frac{a_{H}}{a} \exp \left[-\frac{2}{3}\left(\frac{U_{I}}{U_{H}}\right)^{3 / 2} \frac{a_{H}}{a}\right],
$$


where $U_{H}$ is the ionization potential of hydrogen, $\Omega_{0} \doteq \alpha c / r_{\mathrm{Bohr}} \simeq 4.1 \times 10^{16} \mathrm{sec}^{-1}$ is the "atomic frequency," and $a_{H} \simeq 3.05 \times 10^{14} / \omega\left[\mathrm{sec}^{-1}\right]$ the normalized vector potential associated with the hydrogenic electric field. For circular polarization in which $|\mathbf{E}|$ is constant, Eq. (4) is applicable, while for linear polarization, the cycle averaged form of the tunneling formula should be used,

$$
w(a) \simeq 4 \sqrt{\frac{3}{\pi}} \Omega_{0}\left(\frac{U_{I}}{U_{H}}\right)^{7 / 4} \sqrt{\frac{a_{H}}{a}} \exp \left[-\frac{2}{3}\left(\frac{U_{I}}{U_{H}}\right)^{3 / 2} \frac{a_{H}}{a}\right],
$$

resulting in a slightly higher threshold for rapid ionization in the parameter region of interest. For the purposes of this investigation, in the small region between the multi-photon and tunneling regimes a simple linear interpolation between Eqs. (3) and (4) is used. For $a \sim m_{e}|e|^{6} / \hbar^{4} \omega c$ (beyond the validity range of the Keldysh model), the ionization rate is so rapid that all of the available gas may be treated as ionized instantaneously on the time scale of the laser period.

The composite ionization probability as a function of laser wavelength $\lambda$ and amplitude $a$ for atomic hydrogen and circularly polarized light is plotted in Fig. 1. A window of low ionization probability $\left(w / \omega \leq 10^{-10}\right)$, suitable for propagating the pumping beam over some distance, is evident for $a \leq 0.002$ and $\lambda \geq 0.2 \mu \mathrm{m}$ (corresponding to $I \sim 10^{13} \mathrm{~W} / \mathrm{cm}^{2}$ ), and threshold behavior is seen near $a \simeq 0.002$ over the span of wavelengths $\lambda=0.2-1.0 \mu \mathrm{m}$. Above $a \simeq 0.005\left(I \sim 10^{14} \mathrm{~W} / \mathrm{cm}^{2}\right)$, the ionization rate is effectively instantaneous for $\lambda=0.2-1.0 \mu \mathrm{m}$ marking a lower limit for the seed amplitude in a self-ionizing amplifier. These thresholds are on the order of the values cited for helium in Ref. [21].

Due to photoionization, the electron and neutral densities evolve according to the equations

$$
\partial_{t} n_{e}=n_{n} w(a) \quad \text { and } \quad \partial_{t} n_{n}=-n_{n} w(a) .
$$

By energy conservation, the wave fields must also damp according to

$$
n_{c} \frac{m_{e} c^{2}}{2} \partial_{t}|a|^{2}=-\left(U_{I}+\langle\varepsilon\rangle\right) \partial_{t} n_{e}
$$

where

$$
\frac{E_{1}^{2}+E_{2}^{2}+E_{3}^{2}}{8 \pi}=n_{c} \frac{m_{e} c^{2}}{2}|a|^{2}
$$


is the total electric field energy density, $|a|^{2} \doteq a_{1}^{2}+a_{2}^{2}+\frac{1}{2} \sqrt{\frac{n_{c}}{n_{c}}} a_{3}^{2}$, and $\langle\varepsilon\rangle$ represents the average energy acquired by an electron born in the laser field of magnitude $a(x, t)$. This equation corresponds to the "non-adiabatic" losses due to ionization as discussed in Ref. [22]. For multi-electron atoms, the effect of second and third, etc. ionizations results in additional damping. The far slower process of recombination is completely negligible for the short time scales applicable to Raman amplification. Scattering to other wavelengths due to ionization is also neglected.

The average electron birth energy is given by

$$
\langle\varepsilon\rangle \doteq \frac{1}{N} \int_{0}^{\infty} d \varepsilon f(\varepsilon) \varepsilon \quad \text { with } \quad N \doteq \int_{0}^{\infty} d \varepsilon f(\varepsilon)
$$

where $f(\varepsilon)$ is the electron distribution function in the wake of the ionizing pulse corresponding to electron birth in the given total electric field $a(x, t)$. Note that the instantaneous electric field $a(x, t)$ at the position and time of an electron's birth determines its contribution to the residual oscillation energy $\langle\varepsilon\rangle$. Though the electron oscillates in any subsequent field, only the drift energy imparted to it at the time of ionization (determined by the phase of the electric field) remains with the electron and contributes to the ionization damping of the laser field.

For the case of linear polarization, using the conservation of each electron's transverse canonical momentum $\mathbf{p}-|e| \mathbf{A} / c$ with $\mathbf{A}$ the laser vector potential, the energy distribution of electrons left behind by an ionizing laser pulse (considering only the simple case of hydrogen from which one electron is liberated) may be calculated [23] from Eq. (4) to be

$$
\begin{aligned}
f(\varepsilon)= & \frac{1}{\varepsilon_{\mathrm{osc}}}\left(1-\frac{\varepsilon}{\varepsilon_{\mathrm{osc}}}\right)^{-1}\left(\frac{\varepsilon}{\varepsilon_{\mathrm{osc}}}\right)^{-1 / 2} \times \\
& \exp \left[-\beta\left(1-\frac{\varepsilon}{\varepsilon_{\mathrm{osc}}}\right)^{-1 / 2}\right] \Theta\left(2 \varepsilon_{\mathrm{osc}}-\varepsilon\right),
\end{aligned}
$$

where $\varepsilon_{\text {osc }} \doteq m_{e} c^{2} a^{2} / 4$ is the time averaged oscillation energy of the laser field at the time of ionization, $\beta \doteq(2 / 3)\left(U_{I} / U_{H}\right)^{3 / 2}\left(a_{H} / a\right)$, and $\Theta(x)$ is the unit step function. The distribution is peaked at $\varepsilon=0$ and cutoff at twice the average laser oscillation energy. Physically, noting that Eq. (4) is a strongly 
increasing function of the electric field magnitude, the majority of particles are born at the peaks of the electric field where $\mathbf{A}=\mathbf{0}$. Treating the electrons as born with zero momentum, conservation of the canonical transverse momentum then stipulates that the majority of electrons returns to rest after the passing of the pulse, effectively returning the particle kinetic energy to the pulse, and leaving a distribution peaked at $\varepsilon=0$. Similarly, an electron born in phase with the laser field gains at most $2 \varepsilon_{\text {osc }}$ resulting in the cut-off of the distribution on this collisionless time-scale.

For atomic hydrogen with $U_{I} \simeq 13.6 \mathrm{eV}, \lambda \simeq 0.5 \mu \mathrm{m}$, and $a \sim 0.01-0.001$, then $\beta \sim 5-50 \gg 1$ and the integral may be evaluated asymptotically to yield

$$
\langle\varepsilon\rangle \sim \sqrt{2 \pi} \frac{\varepsilon_{\mathrm{osc}}}{K_{0}(\beta)} \beta^{-3 / 2} e^{-\beta} \sim \frac{m_{e} v_{\mathrm{osc}}^{2}}{2 \beta}, \beta \rightarrow \infty,
$$

where use is made of the large argument approximation for the modified Bessel function $K_{0}(\beta) \sim$ $\sqrt{\pi / 2 \beta} e^{-\beta}$ [24]. At sufficiently large $a, \beta$ is no longer large and the approximation of Eq. (8) no longer applies; however, this results only in an over-estimate of $\langle\varepsilon\rangle$ and, at worst, a conservative estimate of the cost of the ionization damping.

In contrast, for circular polarization, the constancy of the magnitude of the electric field results in a distribution peaked near the laser oscillation energy [25], so that $\langle\varepsilon\rangle \sim m_{e} v_{\mathrm{osc}}^{2} / 2$. Consequently, for large $\beta$, linear polarization results in a substantially reduced contribution of the oscillation energy to the ionization damping rate relative to circular polarization.

\section{Energetics of self-ionizing Raman amplification}

With these equations, an estimate of the overall limitations placed on Raman amplification due to ionization damping may be made. Assuming the seed pulse reaches the advanced nonlinear phase of amplification when significant depletion of the pump occurs, during a time interval $\Delta t$, the seed would 
gain an energy increment from the pump of

$$
\Delta \varepsilon_{+} \sim 2 c \Delta t \epsilon \frac{E_{1}^{2}}{8 \pi} \sim 2 c \Delta t \epsilon n_{c} \frac{m_{e} c^{2}}{2} a_{1}^{2}
$$

where $\epsilon \sim 0.8$ represents the fraction of pump depletion. Simultaneously, the seed would lose an energy increment due to total ionization of the background gas of

$$
\Delta \varepsilon_{-} \sim c \Delta t n_{e}\left(U_{I}+\langle\varepsilon\rangle\right)
$$

so that the criterion for net amplification, $\Delta \varepsilon_{+}>\Delta \varepsilon_{-}$, may be written as

$$
a_{1}>\sqrt{\frac{n_{e}}{\epsilon n_{c}} \frac{U_{I}+\langle\varepsilon\rangle}{m_{e} c^{2}}} .
$$

An accurate estimate of the limitation placed on $a_{1}$ by Eq. (9), requires an evaluation of the average electron birth energy $\langle\varepsilon\rangle$ including two important effects: Foremost, an estimate of the characteristic amplitude in the seed pulse profile at which the majority of electrons are born must be made since the source of electrons $n_{n}$ may be completely depleted long before the the complete seed pulse passes any representative neutral atom. Electrons born in the leading part of the pulse oscillate in the high intensity fields near the peak of the seed, but ultimately return their oscillation energy to the pulse as it passes and retain only the drift energy established by the low intensity field at which they were born. Secondly, as shown for linearly polarized lasers, the majority of particles do not possess the full laser field oscillation velocity corresponding to the field amplitude at which they are born.

The amplitude of $a_{2}$ where the majority of electrons are born (i.e., where $n_{n}$ drops rapidly in the ionizing field of the seed) may be estimated by considering the simple model (valid in the small neighborhood of the steep seed pulse front where most ionization occurs) of a laser pulse whose amplitude increases linearly with a slope $\eta$ behind the pulse front, $a(x, t)=\eta(c t-x) \Theta(c t-x)$, impinging on a step-function distribution of neutral gas, $n_{n}(x, t=0)=\Theta(x)$. For the case of linear polarization, integrating the second of Eqs. (5) for these conditions using the cycle averaged form of Eq. (4) and assuming $a(x, t)$ to 
be unperturbed by the ionization process (i.e., the case of a strong seed in a tenuous gas) gives

$$
\begin{aligned}
n_{n}(x, t)= & \Theta(x) \times \\
& \exp \left\{-\frac{w_{0} \sqrt{\beta^{\prime}}}{c \eta} \Gamma\left[-\frac{1}{2}, \frac{\beta^{\prime}}{\eta(c t-x)}\right] \Theta(c t-x)\right\}
\end{aligned}
$$

where $\beta^{\prime} \doteq(2 / 3)\left(U_{I} / U_{H}\right)^{3 / 2} a_{H}, w_{0} \doteq 4 \sqrt{3 / \pi} \Omega_{0}\left(U_{I} / U_{H}\right)^{7 / 4} \sqrt{a_{H}}$, and $\Gamma(a, x)$ is the incomplete Gamma function. Using the large argument expansion for $\Gamma(a, x)$ [24], in the region just behind the pulse front $\left(\beta^{\prime} / \eta(c t-x) \rightarrow+\infty\right)$,

$$
\begin{aligned}
n_{n}(x, t) \sim & \Theta(x) \times \\
& \exp \left\{-\frac{w_{0} \sqrt{\eta}}{c \beta^{\prime}}(c t-x)^{3 / 2} \exp \left[-\frac{\beta^{\prime}}{\eta(c t-x)}\right]\right\} .
\end{aligned}
$$

For typical parameters, the characteristic depth into the pulse at which the majority of electrons is born is then approximately found to be

$$
(c t-x) \simeq \frac{\beta^{\prime} / \eta}{\ln \left(w_{0} \sqrt{\beta^{\prime}} / c \eta\right)}
$$

so that the corresponding laser intensity in this linear model is then

$$
a \simeq \frac{\beta^{\prime}}{\ln \left(w_{0} \sqrt{\beta^{\prime}} / c \eta\right)} .
$$

In the case of circular polarization, using the unaveraged form of Eq. (4), a similar calculation (with now $\left.w_{0} \doteq 4 \Omega_{0}\left(U_{I} / U_{H}\right)^{5 / 2} a_{H}\right)$ yields for the characteristic field strength at which most electrons are born

$$
a \simeq \frac{\beta^{\prime}}{\ln \left(w_{0} / c \eta\right)}
$$

In both cases, $a$ increases with increasing pulse steepness. Note that, for these parameters, since the cycle-averaged ionization rate appropriate for linear polarization is slightly less than the unaveraged result applicable to circular polarization, the majority of electrons are born at a slightly higher field for linear polarization as opposed to circular polarization. However, the reduction in $\langle\varepsilon\rangle$ for linear polarization largely compensates this effect, so that the characteristic energy at which most electrons 
are born in a linearly polarized pulse is actually less than that for circular polarization for all values of $\eta \lambda$ for which the envelope approximation is valid.

For a typical seed pulse front with $\eta \lambda \simeq 0.1$ in the nonlinear regime and again for the case of atomic hydrogen with $\lambda=0.5 \mu \mathrm{m}$ and $n_{e}=0.001 n_{c}$, using these estimates of $a$ in evaluating Eq. (8) (noting that $\beta$ depends on $a$ for linear polarization) and Eq. (9) leads to

$$
a_{1} \geq \sqrt{\frac{n_{e}}{\epsilon n_{c}} \frac{U_{I}+\langle\varepsilon\rangle}{m_{e} c^{2}}} \simeq 2.2 \times 10^{-4} .
$$

For circular polarization, the amplification condition is

$$
a_{1} \geq 3.1 \times 10^{-4}
$$

for the same parameters. In both cases, a threshold amplitude for the pump must be exceeded to overcome ionization damping. The attainable amplitude of $a_{2}$ is constrained only due to steepening of the pulse front during the amplification process and consequent increase of the characteristic $\langle\varepsilon\rangle$. Such a limit is generally reached only beyond the validity of Eqs. (1), however. Also, though for fixed $a,\langle\varepsilon\rangle$ may be significantly greater for circular polarization, the net advantage of linear polarization in terms of the ionization damping rate proves only marginal due to the dominating contribution of the ionization potential and since most electrons are born at a lower field amplitude with circular polarization for typically steep pulses.

Alternately, with $a_{1}$ constrained by the ionization threshold given in Fig. 1 ( $\left.a_{1} \sim 0.0015\right)$, Eq. (9) constitutes a limitation on the acceptable gas density. Note that, should the allowable pump intensity prove to be lower than that given by Fig. 1 and assumed above, this should only result in the necessity of using lower gas densities in accordance with Eq. (9). However, $n_{n}$ may not be reduced arbitrarily since the resultant plasma must be sufficiently dense to prevent the breaking of the backscatter-driven Langmuir wave and subsequent incomplete pump depletion. Using the wave breaking criterion $\omega_{p}>$ $\omega_{b} \doteq \sqrt{|e| k_{3} E_{3} / m_{e}}$ and the Manely-Rowe relation $a_{1}^{\max } \sim a_{3}^{\max }$ with $k_{3} \simeq 2 \omega / c$ gives $a_{1} \leq\left(\omega_{p} / \omega\right)^{3 / 2} / \sqrt{2}$ 
or $n_{n} / n_{c} \geq 2^{3 / 2} a_{1}^{4 / 3}$ to avoid breaking. The composite criterion on the neutral density may then be written

$$
\begin{aligned}
2^{3 / 2} a_{1}^{4 / 3} \simeq 0.00027<\frac{n_{n}}{n_{c}} & <\epsilon \frac{m_{e} c^{2}}{U_{I}+\langle\varepsilon\rangle} a_{1}^{2} \\
& \simeq \begin{cases}0.048, & \text { linear polarization } \\
0.023, & \text { circular polarization }\end{cases}
\end{aligned}
$$

To within the order unity accuracy to be expected of the above estimates, these limits were observed to be generally consistent with results found numerically (see Sec. 4). For circular and linear polarizations, peak seed amplitudes were observed to stabilized at little more than their initial amplitudes $a_{2} \sim 0.01$ (although pulse shapes would sharply steepen as an effect of the ionization front) for $n_{n}=0.01 n_{c}$. A gas density of $n_{n} \leq 0.002 n_{c}$ was found necessary for substantial amplification of the seed to $a_{2} \geq 0.1$.

A threshold also applies for the amplitude and steepness of the seed pulse for a self-ionizing amplifier to be feasible. In contrast to the pump, constraints on the seed arise from the complex spatiotemporal evolution of an initial pulse through the linear pulse broadening regime into the nonlinear pump depletion regime, including ionization damping, and do not appear amenable to simple arguments of the type used to estimate the pump threshold. The initial seed pulse must be sufficiently intense and the linear Raman growth rate (dependent on $a_{1}$ and $n_{e}$ ) sufficiently rapid that the linear response, growing exponentially in the fully ionized plasma behind the seed, replenishes the seed pulse before the seed intensity is damped below the threshold for rapid ionization. Neglecting ionization damping, the condition for the seed to access the nonlinear regime can be quantified as the seed pulse length $L_{\text {seed }}$ exceeding the Raman growth length in the seed $c / a_{2} \sqrt{\omega \omega_{p} / 2}$ or $a_{2}>\sqrt{2 n_{c} / n_{n}} /\left(k L_{\text {seed }}\right)$. For a $1 \mathrm{ps}$ initial seed, a minimum seed intensity (i.e., ignoring ionization) is then $a_{2}>0.002$ for the same parameters as above.

Numerically, at the threshold density of $n_{e}=0.01 n_{c}$, seed amplitudes of $a_{2} \geq 0.008$ and 0.01 for circular and linear polarizations respectively (comparable to the requirement for rapid ionization) were found 
necessary merely to ensure sustainment of the seed at its initial intensity in the face of ionization. In this regime of relatively gentle pulse fronts where the ionization damping rate for linear polarization slightly exceeds that for circular polarization and since the wave coupling constant $K$ and hence linear Raman growth rate is slightly larger for circular polarization, circular polarization is evidently favored. Similarly, and since linear polarization results in only a slight reduction in ionization damping for the nonlinear regime, circularly polarized fields are also seen to reach slightly higher final amplitudes. In addition, the collisional damping rate for circularly polarized pulses is less than that for linear polarization due to the presence of stagnation points in electron orbits in linearly polarized fields.

Combining the first of Eqs. (5) with Eq. (6) shows that the wave energy damping from ionization scales as $n_{n} / n_{c}$. Since the linear Raman growth rate scales as $a_{1} K \sim a_{1}\left(n_{e} / n_{c}\right)^{1 / 4} \sim a_{1}\left(n_{n} / n_{c}\right)^{1 / 4}$, for any fixed $a_{1}$, the growth rate during the crucial linear phase may then always be arranged to exceed the ionization damping by sufficiently reducing $n_{n}$. Reducing the linear wave coupling incurs the cost of a longer interaction length to access the nonlinear regime; however, the only limit to this process is that imposed by the physically allowable size of a practical device and associated optics.

In the conventional Raman amplification scheme, the total amplification process is expected to be limited by either Raman forward scattering or modulational instabilities of the highly amplified seed to times less than $t_{\mathrm{amp}} \sim 1 / \omega_{p} a_{1}^{4 / 3}$. Maximizing $t_{\mathrm{amp}}$ by minimizing $\omega_{p}$ subject to the constraint that $\omega_{p} \geq \omega\left(4 a_{1}\right)^{2 / 3}$ to avoid Langmuir wave-breaking and incomplete pump depletion gives a maximum amplification length of $L_{\mathrm{amp}} \sim \lambda / a_{1}^{2}$. Also using the limit $\omega_{p} \sim \omega\left(4 a_{1}\right)^{2 / 3}$, the output pulse temporal length is of the order of $T_{\text {out }}[\mathrm{fs}] \sim 6 / \omega_{p} \sim 1.3 \lambda[\mu \mathrm{m}] / a_{1}^{2 / 3}$, so that the output pulse peak intensity for linear polarization is $I_{\text {out }}\left[\mathrm{W} / \mathrm{cm}^{2}\right] \sim \epsilon L_{\mathrm{amp}} E_{1}^{2} / 8 \pi c T_{\text {out }} \sim 4 \times 10^{10} \epsilon a_{1}^{2 / 3} / \lambda[\mathrm{cm}]^{2}$ and its amplitude $a_{2, \mathrm{out}} \sim\left(I \lambda^{2}[\mathrm{~W}] / 1.37 \times\right.$ $\left.10^{10}\right)^{1 / 2} \sim 1.7 \epsilon^{1 / 2} a_{1}^{1 / 3}$. As shown above, self-ionization, in addition to constraining the pump to intensities less than the threshold for significant ionization, only places a criterion on the minimum pump intensity. In the region of parameter space where these two constraints are compatible, and 
assuming self-ionizing amplification to be limited by forward Raman or modulational instabilities as in the conventional case, the output pulse parameters may be calculated by the above formulas. Example parameters for a self-ionizing amplifier using atomic hydrogen with $a_{1}=0.0015$ are summarized in Table 1. All appear to be within the constraints of a practically realizable device.

Table 1: Example output parameters.

\begin{tabular}{llll}
$\lambda[\mu \mathrm{m}]$ & 0.2 & 0.5 & 1.0 \\
\cline { 2 - 4 }$n_{e} / n_{c}$ & 0.001 & 0.001 & 0.001 \\
$L_{\mathrm{amp}}[\mathrm{cm}] \sim \lambda[\mathrm{cm}] / 4 a_{1}^{2}$ & 2.22 & 5.55 & 11.1 \\
$a_{2, \text { out }} \sim 1.2 a_{1}^{1 / 3}$ & 0.14 & 0.14 & 0.14 \\
$T_{\text {out }}[\mathrm{fs}] \sim 1.3 \lambda[\mu \mathrm{m}] / a_{1}^{2 / 3}$ & 49.6 & 124 & 248 \\
$I_{\text {out }}\left[\mathrm{W} / \mathrm{cm}^{2}\right] \sim 4 \times 10^{10} \epsilon a_{1}^{2 / 3} / \lambda[\mathrm{cm}]^{2}$ & $1.3 \times 10^{18}$ & $2.1 \times 10^{17}$ & $5.2 \times 10^{16}$
\end{tabular}

The above calculations have been for the simple, mono-electronic case of hydrogen. The somewhat higher first ionization potential of helium, however, makes this gas more desirable as an amplifying medium since it could sustain higher pump intensities with ionization, but its second electron complicates the energy arguments given above for hydrogen. Simply approximating the oscillation energies $\left\langle\varepsilon_{i}\right\rangle$ of each electron liberated from helium as being of the order of the corresponding ionization potential $U_{i}$ and since helium can with stand pump intensities of $a_{1} \sim 0.002$ without ionizing leads to the conditions

$$
\begin{gathered}
\frac{n_{n}}{n_{c}}<\epsilon \frac{m_{e} c^{2}}{2\left(U_{1}+U_{2}\right)} a_{1}^{2} \sim 0.0052 \\
L_{\mathrm{amp}} \sim \frac{\lambda}{4 a_{1}^{2}} \sim 3.13 \mathrm{~cm} \\
I_{\text {out }} \sim 4 \times 10^{10} \epsilon \frac{a_{1}^{2 / 3}}{\lambda^{2}} \sim 2.5 \times 10^{17} \mathrm{~W} / \mathrm{cm}^{2}
\end{gathered}
$$

for $\lambda=0.5 \mu \mathrm{m}$. Since no gas has a higher first ionization potential than helium, a helium medium permits the highest pump intensities for a self-ionizing amplifier and hence accesses the limiting intensity of $\sim 10^{17} \mathrm{~W} / \mathrm{cm}^{2}$ for $0.5 \mu \mathrm{m}$ radiation in the shortest possible distance. 


\section{Example simulation of self-ionizing amplification}

With the assumptions discussed above, Eqs. (1), (2), (5), and (6) have been integrated numerically in the frame of the seed pulse. Fig. (2) gives an example comparison of the time evolution of identical seed pulses of $a_{2}(t=0)=0.01$ and $\lambda=0.5 \mu \mathrm{m}$ amplified by a pump of $a_{1}=0.0015$ in a pre-ionized plasma of $n_{e}=0.001 n_{c}$ and $T_{e}=500 \mathrm{eV}$ and in an ionizing atomic hydrogen gas of the same density. In both cases, the lasers are circularly polarized.

In the pre-ionized case, the characteristic " $\pi$-pulse" shape of the nonlinear regime of amplification is evident subject to modifications due to slight collisional damping. The peak amplitude of the leading spike is seen to grow linearly with time, while the pulse width decreases inversely with time. A total amplification by a factor of $\sim 12$, corresponding to an output intensity of $1.6 \times 10^{17} \mathrm{~W} / \mathrm{cm}^{2}$, is reached for the leading spike with an output pulse width equivalent to one half of the input width. Beyond $a_{2} \sim 0.14$, a modulational instability of the high-amplitude pulse is expected to end the amplification process and invalidate the approximations of Eqs. (1).

Remarkably, the self-ionizing pulse reaches a slightly higher amplitude and is slightly more compressed but is seen also to have fallen behind its analogue for the pre-ionized case. This results from the fact that during the early phase of amplification, the leading edge of the relatively low amplitude pulse only partially ionizes the gas (and so is not amplified) but yet undergoes significant ionization damping. This remnant of the original seed is evident as the low amplitude foot stretching ahead of the main peak in an only very weakly ionized plasma. Simultaneously, the later segments of the pulse are strongly amplified in a fully ionized plasma with low damping and so result in an effective shift of the pulse peak to the right. Note that, though not evident in Fig. (2), both pulses evolve towards the $\pi$-pulse through similar linear regimes: a pulse broadening regime characteristic of the linearized solutions of Eqs. (1) [8], with the addition of a notching of the initial seed pulse due to ionization damping in the ionizing case. That both reach similar asymptotic states is evidence of the $\pi$-pulse remaining the attractor solution 
of Eqs. (1) despite the substantial perturbation introduced by ionization effects.

\section{Conclusions}

In summary, an elementary analysis has demonstrated a regime of feasibility for a self-ionizing, plasmabased backward Raman amplifier. In the conventional Raman amplifier, instability of the pumping beam to spontaneous backscatter due to fluctuations in the pre-formed plasma medium may be a severe obstacle to successful amplification. By using the intense amplifying seed pulse to generate the plasma by photoionization of a neutral gas only in the region where Raman scattering is needed to occur, the instability of the pump may be avoided provided that the pump intensity is kept below the threshold for significant ionization. It has been shown that the damping rate due to photoionization by the seed may be successfully overcome by the non-linear Raman amplification rate and that this ionization damping results only in the requirement of a minimum pump intensity which is consistent with a pump intensity below the threshold of photoionization provided the working gas is sufficiently rarefied. A sufficiently intense seed is also required to photoionize the gas rapidly and to access the nonlinear regime of Raman amplification before excessive ionization damping occurs. The output parameters of a self-ionizing Raman amplifier are then expected to scale in an equivalent manner to those of the conventional Raman amplifier operated with the same pump intensity. Though the detailed kinetics of ionization in linearly and circularly polarized fields are quite different, only marginal net differences are found between these polarizations for Raman amplification, with circular polarization appearing slightly favored.

Note that, in addition to the Raman pulse compression regime considered here, a similar effect might accompany pulse compression in the so-called Compton scattering regime, where backscattering might occur due to electron trapping effects rather than through a resonant three-wave interaction [26]. The self-ionizing backscatter effect, if it occurs for the Compton regime, could then be addressed by an 
approach similar to the one employed here for the Raman regime.

The preceding analysis has entailed many simplifications, however. Foremost, while self-ionization avoids instabilities resulting from the propagation of the pump beam through plasma, instabilities which might be introduced due to the sharp ionization front have been entirely neglected in this analysis. Specifically, an instability involving transverse modulations of the ionization front due to the nonlinearity of Eq. (4) has been identified [27]. If the exponentiation length for such an instability proves to be less than that of the relativistic modulational instability cited above, then a new limiting constraint on the total allowable amplification length (and hence output intensity) applies. However, since this instability depends only on the amplitude of the foot of the seed pulse (where ionization occurs) and not on the peak seed amplitude (as is the case for the relativistic modulational instability), the peak output intensity is not constrained by this instability. Simply initializing the seed with a higher intensity (perhaps from a previous Raman amplifier) and amplifying it over a shorter length would then result in the same output power as if a smaller initial seed were amplified over a longer length in the absence of the instability. The complicating effects of harmonic generation [28], ionization-induced blue-shifting [29, 30], or forward Raman scattering in an ionizing plasma have similarly not been included.

Further simplifications include that the Keldysh ionization model represents a much reduced analytical approximation to a complex physical phenomena. Using a simple interpolation formula to connect the multiphoton and tunneling ionization regimes is a further lowest-order approximation. The effect of collisional ionization (negligible for the short seed pulse, but possibly important for the long pump pulse) has also been neglected. Particularly, over-estimating the maximum allowable pump intensity to avoid ionization or underestimating the minimum permissible seed intensity needed for rapid ionization could alter the results presented above; however, as indicated in Eq. (10), over-estimating the allowable pump intensity should only necessitate using a lower gas density and a longer interaction length for the same output intensity (provided wave breaking is avoided). The validity of the envelope approximation 
to the wave coupling problem as introduced in Eqs. (1) also becomes uncertain when extremely steep gradients in wave intensities, as results from the ionization front, appear. The exact behavior of the electron distribution created by such a rapidly changing pulse may similarly be more subtle than is captured in the ionization damping rate estimate used here. The result cited in Eq. (7) is strictly valid only for the case of adiabatic laser pulse shapes and not those with extremely steep fronts. In treating the sharp ionization front, no attempt was further made to treat realistically the Raman resonance condition for such an inhomogeneous plasma. Possible relativistic effects at very high intensities have also been neglected. Finally, two- or three-dimensional effects associated with finite focusing lengths or transverse plasma or laser field intensity gradients, etc. have not been considered. In principle, all of these deficiencies might be addressed by PIC simulation of a self-ionizing amplifier such as with $[10,31,32]$ in which an ionization package has been included.

\section{Acknowledgments}

The authors gratefully acknowledge E. J. Valeo and S. Brunner for many useful conversations regarding photoionization, particularly in pointing out the work of Corkum, et al. They also thank V. M. Malkin for discussions and Yu. Tsidulko for suggesting the algorithm used in integrating Eqs. (1). This work was supported by the U.S. Department of Energy Contract No. DE-AC02-76-CHO-3073 and by DARPA.

\section{References}

[1] J. D. Lindl, Inertial Confinement Fusion: the Quest for Ignition and Energy Gain Using Indirect Drive, American Institute of Physics, New York, 1998.

[2] E. Esarey, P. Sprangle, J. Krall, and A. Ting, IEEE Trans. Plasma Sci. 24, 252 (1996). 
[3] C. Bula, K. T. McDonald, E. J. Prebys, C. Bamber, S. Boege, T. Kotseroglou, A. C. Melissinos, D. D. Meyerhofer, W. Ragg, D. L. Burke, R. C. Field, G. HortonSmith, A. C. Odian, J. E. Spencer, D. Walz, S. C. Berridge, W. M. Bugg, K. Shmakov, and A. W. Weidemann, Phys. Rev. Lett. 76, 3116 (1996).

[4] G. A. Mourou, C. P. J. Barty, and M. D. Perry, Physics Today 51, 22 (1998).

[5] M. Tabak, J. Hammer, M. E. Glinsky, W. L. Kruer, S. C. Wilks, J. Woodworth, E. M. Campbell, and M. D. Perry, Phys. Plasmas 1, 1626 (1994).

[6] V. M. Malkin, G. Shvets, and N. J. Fisch, Phys. Rev. Lett. 82, 4448 (1999).

[7] V. M. Malkin, G. Shvets, and N. J. Fisch, Phys. Rev. Lett. 84, 1208 (2000).

[8] B. L. Bobroff and H. A. Haus, J. Appl. Phys. 38, 390 (1967).

[9] V. M. Malkin, G. Shvets, and N. J. Fisch, Phys. Plasmas 7, 2232 (2000).

[10] A. B. Langdon and B. F. Lasinski, Electromagnetic and relativistic plasma simulation models, in Methods in Computational Physics, edited by J. Killeen, R. Alder, S. Fernbach, and M. Rotenberg, volume 16, p. 327, New York, 1976, Advances in Research and Applications, Academic Press.

[11] A. B. Langdon, J. Comp. Phys. , 202 (1979).

[12] V. M. Malkin and N. J. Fisch, Backward Raman amplification of ionizing laser pulses, to appear in Phys. Plasmas, 2001.

[13] Y. A. Tsidulko, V. M. Malkin, and N. J. Fisch, Suppression of superluminous precursors in high power backward Raman amplifiers, submitted for publication, 2001.

[14] S. P. Nikitin, Y. Li, T. M. Antonsen, and H. M. Milchberg, Opt. Commun. 157, 139 (1998). 
[15] A. Bers, Linear waves and instabilities, in Plasma Physics, edited by C. DeWitt and J. Peyraud, p. 113, Les Houches, 1972, Université de Grenoble, Summer School of Theoretical Physics, Gordon \& Breach, New York.

[16] W. L. Kruer, The Physics of Laser Plasma Interactions, Addison-Wesley, New York, 1988.

[17] J. D. Huba, editor, NRL Plasma Formulary, Naval Research Laboratory Publication NRL/PU/6790-94-265 (U. S. GPO), Washington, D. C., 1994.

[18] P. Sprangle, E. Esarey, and J. Krall, Phys. Rev. E 54, 4211 (1996).

[19] L. V. Keldysh, Sov. Phys. JETP 20, 1307 (1965).

[20] L. D. Landau and E. M. Lifshitz, Quantum Mechanics: Non-relativistic Theory, Pergamon, Oxford, 1977.

[21] T. P. Hughes, Plasmas and Laser Light, Adam Hilger, Bristol, England, 1975.

[22] N. E. Andreev, M. E. Veisman, M. G. Cadjan, and M. V. Chegotov, Plasma Phys. Reports 26, $947(2000)$

[23] N. H. Burnett and P. B. Corkum, J. Opt. Soc. Am. B 6, 1195 (1989).

[24] M. Abramowitz and I. A. Stegun, Handbook of Mathematical Functions, Wiley, New York, 1984.

[25] P. B. Corkum, N. H. Burnett, and F. Brunel, Phys. Rev. Lett. 62, 1259 (1989).

[26] G. Shvets, N. J. Fisch, A. Pukhov, and J. Meyer-ter-Vehn, Phys. Rev. Lett. 81, 4879 (1998).

[27] T. M. Antonsen and Z. Bian, Phys. Rev. Lett. 82, 3617 (1999).

[28] W. P. Leemans, C. E. Clayton, W. B. Mori, K. A. Marsh, P. K. Kaw, A. Dyson, C. Joshi, and J. M. Wallace, Phys. Rev. A 46, 1091 (1992).

[29] E. Esarey, G. Joyce, and P. Sprangle, Phys. Rev. A 44, 3908 (1991). 
[30] S. C. Rae and K. Burnett, Phys. Rev. A 46, 1084 (1992).

[31] D. F. Gordon, W. B. Mori, and T. M. Antonsen, Jr., IEEE Trans. Plasma Sci. 28, 1224 (2000).

[32] J. P. Verboncoeur, A. B. Langdon, and N. T. Gladd, Computer Phys. Commu. 87, 199 (1995). 


\section{Figure Captions}

Figure1: Ionization probability $w(a, \lambda) / \omega$ for hydrogen.

Figure2: Comparison of pre-ionized (above) and self-ionizing (below) Raman amplification. 
Figure 1:

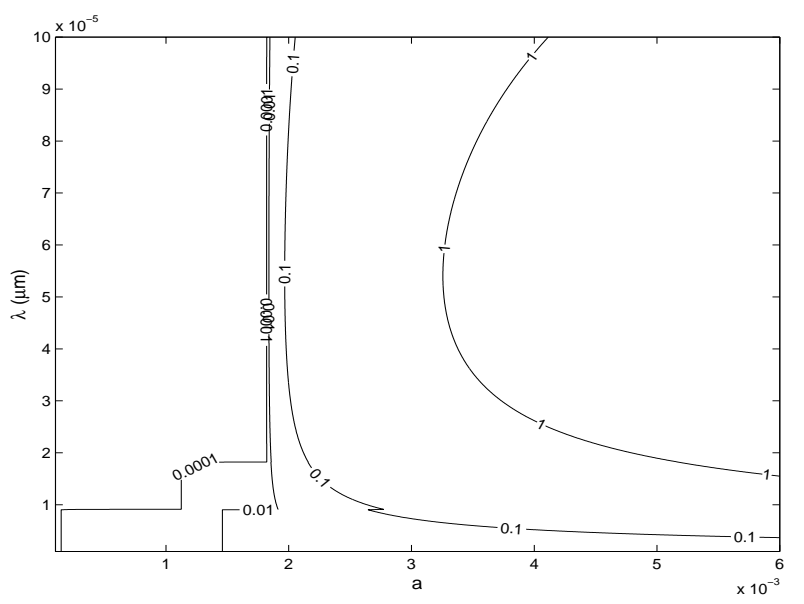


Figure 2:
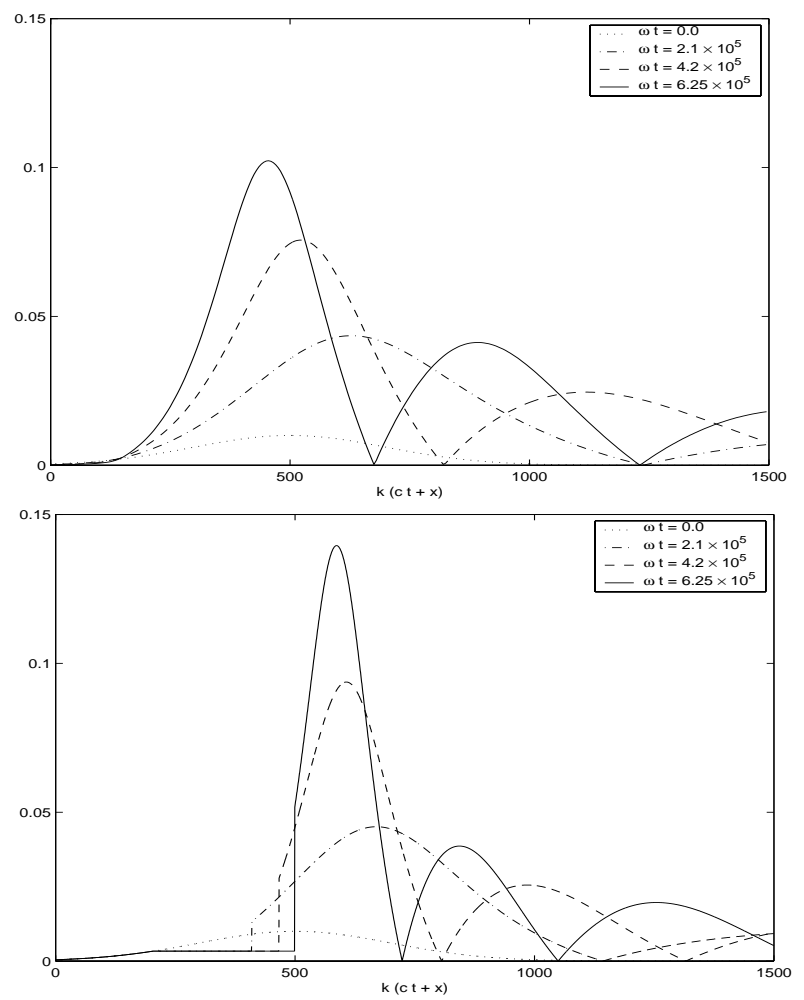


\section{External Distribution}

Plasma Research Laboratory, Australian National University, Australia

Professor I.R. J ones, Flinders University, Australia

Professor J oão Canalle, Instituto de Fisica DEQ/IF - UERJ , Brazil

Mr. Gerson O. Ludwig, Instituto Nacional de Pesquisas, Brazil

Dr. P.H. Sakanaka, Instituto Fisica, Brazil

The Librarian, Culham Laboratory, England

Library, R61, Rutherford Appleton Laboratory, England

Mrs. S.A. Hutchinson, JET Library, England

Professor M.N. Bussac, Ecole Polytechnique, France

Librarian, Max-Planck-Institut für Plasmaphysik, Germany

J olan Moldvai, Reports Library, MTA KFKI-ATKI, Hungary

Dr. P. Kaw, Institute for Plasma Research, India

Ms. P.J . Pathak, Librarian, Insitute for Plasma Research, India

Ms. Clelia De Palo, Associazione EURATOM-ENEA, I taly

Dr. G. Grosso, Instituto di Fisica del Plasma, Italy

Librarian, Naka Fusion Research Establishment, J AERI, J apan

Library, Plasma Physics Laboratory, Kyoto University, J apan

Research Information Center, National Institute for Fusion Science, J apan

Dr. O. Mitarai, Kyushu Tokai University, J apan

Library, Academia Sinica, Institute of Plasma Physics, People's Republic of China

Shih-Tung Tsai, Institute of Physics, Chinese Academy of Sciences, People's Republic of China

Dr. S. Mirnov, TRINITI, Troitsk, Russian Federation, Russia

Dr. V.S. Strelkov, Kurchatov Institute, Russian Federation, Russia

Professor Peter Lukac, Katedra Fyziky Plazmy MFF UK, Mlynska dolina F-2, Komenskeho Univerzita, SK-842 15 Bratislava, Slovakia

Dr. G.S. Lee, Korea Basic Science Institute, South Korea

Mr. Dennis Bruggink, Fusion Library, University of Wisconsin, USA

Institute for Plasma Research, University of Maryland, USA

Librarian, Fusion Energy Division, Oak Ridge National Laboratory, USA

Librarian, Institute of Fusion Studies, University of Texas, USA

Librarian, Magnetic Fusion Program, Lawrence Livermore National Laboratory, USA

Library, General Atomics, USA

Plasma Physics Group, Fusion Energy Research Program, University of California at San Diego, USA

Plasma Physics Library, Columbia University, USA

Alkesh Punjabi, Center for Fusion Research and Training, Hampton University, USA

Dr. W.M. Stacey, Fusion Research Center, Georgia Institute of Technology, USA

Dr. J ohn Willis, U.S. Department of Energy, Office of Fusion Energy Sciences, USA

Mr. Paul H. Wright, Indianapolis, Indiana, USA 
The Princeton Plasma Physics Laboratory is operated by Princeton University under contract with the U.S. Department of Energy.

\author{
Information Services \\ Princeton Plasma Physics Laboratory \\ P.O. Box 451 \\ Princeton, NJ 08543
}

Phone: 609-243-2750

Fax: 609-243-2751

e-mail: pppl_info@pppl.gov

Internet Address: http://www.pppl.gov 